\title{
Exploring Indicators of Hepatotoxicity- Related Post-Marketing Regulatory Actions: A Retrospective Analysis of Drug Approval Data
}

\author{
Salwa M. Almomen ${ }^{\text {a }}$ Mona A. Almaghrabi ${ }^{\text {b }}$ Saja M. Alhabardi ${ }^{b}$ \\ Adel A. Alrwisan ${ }^{a}$

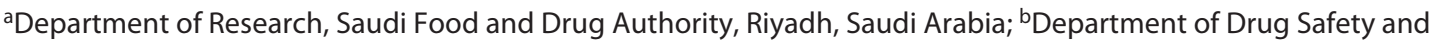 \\ Risk Management, Saudi Food and Drug Authority, Riyadh, Saudi Arabia
}

\section{Keywords}

Post-marketing hepatotoxicity · Predictors · Drug safety

\begin{abstract}
Background: Hepatotoxicity is a major reason for medication withdrawal from the markets. Unfortunately, serious adverse hepatic effects can occur after marketing with limited indicators during clinical development. Therefore, finding possible predictors for hepatotoxicity might guide the monitoring program of various stakeholders such as drug regulatory authorities. Objective: To explore the potential of drugs, pre-approval regulatory factors as predictors for the occurrence of hepatotoxicity-related post-marketing regulatory actions. Pre-approval factors were specified as: (a) Hy's Law hepatotoxicity grade $\geq 3$, (b) accelerated approval status, and (c) labeled hepatic adverse effects and regulatory actions at approval. Methods: Using publicly accessible FDA data, we examined clinical review documents for drugs approved in the USA during the period from 2011 to 2016 to evaluate their hepatic safety profile, identifying the 3 specified indicators for analysis. Predictors (Covariates): We assessed whether these medications meet: (a) Hy's Law hepatotoxicity grade $\geq 3$, (b) accelerated approval status, and (c) labeled hepatic adverse effects and regulatory actions at approval. Outcome (Dependent Variable): Post-marketing
\end{abstract}

regulatory action related to hepatotoxicity including products withdrawal and updates to either warning, precaution or adverse effects sections. Statistical Analysis: Drugs that were approved between 2011 and 2016 were included in the analysis with follow-up time from the date of approval until end of December 2019 or the date of first post-marketing regulatory action related to hepatotoxicity, whichever occurred first. The hazard ratio (HR) was estimated using Coxregression analysis. Results: A total of 192 medications were included in the study. We classified 48 drugs with grade $\geq 3$ hepatotoxicity, 43 with accelerated approval status, and 74 with labeled information about hepatotoxicity prior to marketing. The adjusted HRs for post-marketing regulatory action for products with grade $\geq 3$ hepatotoxicity was 0.61 (95\% confidence interval [Cl], 0.17-2.23), 0.92 (95\% Cl, 0.292.93) for drug approved via accelerated approval program, and 0.91 (95\% Cl, 0.33-2.56) for drugs with labeled hepatotoxicity information at approval time. Conclusion: Hy's Law with hepatotoxicity grade $\geq 3$, accelerated approval, and label information on hepatotoxicity were not identified as predictors for post-marketing additional regulatory actions concerning liver adverse effects. However, the evidence is inconclusive due to small sample size and potential channeling bias.

(c) 2022 The Author(s)

Published by S. Karger AG, Basel karger@karger.com www.karger.com/sjh

Karger $\stackrel{\text { ' }}{5}$

GOPEN ACCESS
C 2022 The Author(s).

Published by S. Karger AG, Basel

This is an Open Access article licensed under the Creative Commons Attribution-NonCommercial-4.0 International License (CC BY-NC) (http://www.karger.com/Services/OpenAccessLicense), applicable to the online version of the article only. Usage and distribution for commercial purposes requires written permission.
Correspondence to:

Salwa M. Almomen, smmomen@sfda.gov.sa 


\section{Introduction}

Drug-induced liver injury (DILI) is defined as a hepatic injury resulting from medications, herbs or any chemical entities, causing abnormal changes to liver tests or liver dysfunction with the reasonable elimination of other possibly offending etiologies [1]. There are various mechanisms for occurrence of DILI, and the resulting injury is similar to roughly all other liver diseases. This contributes to the uncertainty of DILI diagnosis since there are no pathognomonic findings for it, even by liver biopsy [2].

The leading cause of acute liver failure in the USA is attributed to DILI, which accounts for $13 \%$ of the cases, posing a constant challenge for drug development and safety [1]. In fact, DILI is the most common cited cause of safety-related withdrawal of medications from the market for the past 50 years [2-4].

Unfortunately, the detection of DILI before drug approval may be overlooked, due to relatively small sample size of premarketing clinical trials. The incidence of missed cases of DILI is estimated to be 1 in 10,000 [5]. Additionally, in every 10 cases of liver enzymes elevation of 10 times over the upper limit of normal in a clinical trial, 1 case of liver injury with higher severity is expected to occur once the drug becomes available in the markets [5]. Therefore, it is essential to explore possible predictors of hepatotoxicity as a measure to focus the post-marketing drug surveillance programs.

Safety-related information on medications undergoing FDA approval steps may be useful as indicators of future post-approval regulation that may be expected to occur after the drugs are marketed and used. Examples of safety-related information are measures of liver toxicity such as Hy's Law scoring of hepatic injury, accelerated approval programs granting fast-access to critical drugs on demand and information regarding adverse events of medications that are mostly labeled in medication information leaflets.

In this study, we explore the potential of the safetyrelated information described above as predictors for regulatory actions related to hepatotoxicity after approval. Our goal is to guide policy makers in considerations that could be taken regarding post-marketing hepatotoxicityrelated regulatory actions.

\section{Methods}

Data Source and Inclusion Criteria

We searched the United States Food and Drug Administration's website to identify new chemical entities or biological prod- ucts approved from 2011 to 2016 . The reason for limiting the study years up to 2016 is to allow a reasonable time from noting evidence for hepatotoxicity and regulatory action, if any. Information about hepatotoxicity at the time of approval was extracted from the FDA's medical reviews documents. Collection of post-marketing regulatory actions information was through search the FDA's website. The data collection has ended by December 2019 and extracted by 2 independent reviewers.

\section{Outcome}

The definition of post-marketing regulatory action due to hepatotoxicity was inclusive of product withdrawal and updates to the warnings, precautions, or adverse effects related to liver injury.

\section{Covariates}

The indicators used in assessing the risk of post-approval regulatory action resulting from hepatic adverse effects were (1) grade 3 or above hepatotoxicity based on Hy's Law (liver enzymes level of $>5.0-20$ and bilirubin level of $>3.0-10)[6,7]$, (2) whether the drug was approved through the accelerated approvals program, and (3) availability of information about hepatotoxicity in the product's label at approval time.

\section{Hy's Law}

In the analysis of drugs predictors for post-marketing additional regulatory actions concerning hepatic adverse effects, the choice of Hy's Law hepatotoxicity grade $\geq 3$ as the cutoff point is based on the consideration of 2 factors; (a) clinical relevance of drug-induced liver chemistry abnormalities (toxicity severity level) and (b) elevated liver enzymes levels proposed as predictors of toxicity likelihood in the literature [8]. Hepatotoxicity grade 3 describes liver abnormalities of a serious level showing functional effect such as symptoms that could disable patients from work or require hospitalization for close monitoring. The likelihood of hepatocellular drug-induced injury increases with high clinical importance starting at hepatotoxicity grade 3 [8]. The FDA's guidance highlighted that extreme liver enzymes elevations may be better predictors of toxicity than smaller elevations, especially that normalization of liver abnormalities on continued medication use can occur [9]. We hypothesized that occurrence of hepatic adverse effects that leads to issuing additional regulatory actions is more likely with high toxicity levels. Therefore, hepatotoxicity severity level of grade 3 and above was the cutoff line selected as a predictor in this study.

\section{Accelerated Approval}

Accelerated approvals indicator was selected as a covariate considering that drugs approved through FDA Accelerated Approval Program are more likely to have post-marketing regulatory action for safety concerns $[10,11]$.

\section{Statistical Analysis}

The follow-up period for each medication started from the approval date to announcement date of first post-marketing regulatory actions or end of December 2019, whichever came first. Hazard ratios (HRs) and 95\% confidence intervals (95\% CI) were calculated for the 3 defined covariates to explore their association with post-marketing hepatotoxicity-related regulatory actions using Cox-regression model.
Almomen/Almaghrabi/Alhabardi/ Alrwisan 
Table 1. Description of included drugs

\begin{tabular}{llll}
\hline Accelerated approval, $n$ (\%) & No $=149(77.6)$ & & $\begin{array}{l}\text { Average follow-up } \\
\text { time, days }\end{array}$ \\
& Yes $=43(22.4)$ & $38(19.7)$ & $1,855.5$ \\
Therapeutic group, $n$ (\%) & Nervous system & $17(8.8)$ & $2,027.6$ \\
& Anti-infection medications & $7(3.6)$ & $2,002.9$ \\
& Antibiotics & $6(3.1)$ & $1,912.2$ \\
& Anticoagulation medications & $10(5.2)$ & $2,153.9$ \\
& Cardiovascular system medications & $9(4.7)$ & $2,127.9$ \\
& Anti-diabetes medications & $39(20)$ & $2,116.6$ \\
& Anti-neoplastic medications & $9(4.7)$ & $2,312.4$ \\
& Anti-psychotic medications & $5(2.6)$ & $2,646.6$ \\
& Respiratory system medications & $52(27)$ & $2,140.2$ \\
\hline Aarious & $28(13.9)$ & \\
& 2011 & $39(19.3)$ & \\
& 2012 & $27(13.4)$ & \\
& 2013 & $41(20.3)$ & \\
\hline
\end{tabular}

Table 2. Predictors of post-marketing regulatory action related to hepatic adverse effects

\begin{tabular}{|c|c|c|c|c|}
\hline Covariate* & Frequency (\%) & Regulatory action, $n(\%)$ & Adj. HR** & $95 \% \mathrm{Cl}$ \\
\hline \multicolumn{5}{|c|}{ Hepatotoxicity grade } \\
\hline$<3$ (reference) & $144(75)$ & $3(1.6)$ & \multirow{2}{*}{0.612} & \multirow{2}{*}{$0.17-2.23$} \\
\hline$\geq 3$ & $48(25)$ & $14(7.3)$ & & \\
\hline \multicolumn{5}{|c|}{ Accelerated approval } \\
\hline No (reference) & $149(77.8)$ & $13(6.8)$ & \multirow{2}{*}{0.92} & \multirow{2}{*}{$0.29-2.93$} \\
\hline Yes & $43(22.4)$ & $4(20.1)$ & & \\
\hline \multicolumn{5}{|l|}{ Action at approval } \\
\hline No (reference) & $118(61.5)$ & $11(5.7)$ & \multirow{2}{*}{0.91} & \multirow{2}{*}{$0.33-2.56$} \\
\hline Yes & $74(36.5)$ & $6(3.1)$ & & \\
\hline
\end{tabular}

Adj. HR, adjusted hazard ratio. * Covariate follow-up time: from date of drug approval to date of first postmarketing regulatory action or end of study timeline (up-to-date: December 31, 2019), whichever comes first. ** HR adjusted for hepatotoxicity grade accelerated approval and action at approval.

\section{Results}

During the period of 2011-2016, a total of 192 drugs were approved by the FDA and classified into 10 therapeutic groups using anatomical therapeutic classification. Drugs included in the study were mostly antineoplastic 39 (20\%) and nervous system agents 38 (19.7\%). Drugs approved through the accelerated approval program accounted for $22.4 \%$ of the study sample. The number of drugs approved in 2015 was the highest across the study years (Table 1).

Post-Marketing Hepatotoxicity Predictors
Seventeen post-approval regulatory actions have occurred during the study period (Table 2). Most of the post-marketing regulatory actions were issued to drugs in the therapeutic group of chemotherapy/oncology/hematology/antineoplastic ( 7 out of 17) followed by drugs in the therapeutic group of immunomodulatory and biological therapy (4 out of 17). One-quarter of the study drugs $(n=48)$ met Hy's Law hepatotoxicity grade $\geq 3$. Fortythree drugs $(22.4 \%)$ were approved through the accelerated approval program, and 74 (36.5\%) drugs had labeled hepatic safety information at approval time (Table 2 ). 
The HR for post-marketing regulatory action for drugs that scored Hy's Law grade $\geq 3$ versus drugs that scored lower Hy's Law grades was 0.61 (95\% CI, 0.17-2.23). Likewise, there was no increase in the HR for post-approval status for drugs approved through the accelerated approval program in comparison to drugs not approved through the program (HR, 0.92; 95\% CI, 0.29-2.93). As well, HR for drugs with labeled hepatic safety information was not increased in comparison to drugs with no such labels (HR, 0.91; 95\% CI, 0.33-2.56) (Table 2).

\section{Discussion}

Despite of the efforts to exclude hepatotoxic drugs before they enter clinical trial phase, some drugs escape the preclinical toxicity testing to reach advanced stages of drug development and approval [11].

Our analysis showed that accelerated approval is not associated with post-approval regulatory actions related to hepatotoxicity. On the contrary, a study in 2017 described an association; however, post-approval regulations measurement in that study was not exclusive to hepatotoxicity-related adverse events [12].

In this study, Hy's Law with hepatotoxicity grade $\geq 3$ was not associated with increased hazard for post-marketing regulatory action. However, the literature is replete with examples of post-marketing hepatotoxicityrelated regulatory actions where products have met Hy's Law case identification during drug development program. For instance, 2 Hy's Law cases in 1,000 exposures were identified during pre-approval clinical trials for dilevalol, which later on was withdrawn from Portuguese markets based on cases of fatal liver injuries [2]. Other examples include cases of DILI with the use of bromfenac, troglitazone, and ximelagatran, which had documented Hy's Law cases during clinical trials [2]. We have identified 2 limiting factors that may have played a role in not detecting association of Hy's Law grade $\geq 3$ factor with post-marketing hepatotoxicity-related regulations. First, small sample size $(n=192)$, which is further reflected by the wide CI ( $95 \%$ CI $=0.17-2.23)$. Second, channeling bias may have occurred since drugs with noted higher hepatotoxicity index could be under-prescribed or avoided by physicians that would resort to alternative medications with lower hepatotoxicity index [13]. Consequently, reported side effects could be lower leading to lowered chances of additional regulatory actions occurrence.

\section{Conclusion}

Hy's Law with hepatotoxicity grade $\geq 3$, accelerated approval and label information on hepatotoxicity were not identified as predictors for post-marketing additional regulatory actions concerning liver adverse effects. However, the evidence is inconclusive due to small sample size and potential channeling bias.

\section{Statement of Ethics}

Ethics approval was not required. This decision was approved by The Scientific Ethical Committee at the Department of Research and Studies of the Saudi Food and Drug Authority. Informed consent was not required. This decision was approved by The Scientific Ethical Committee at the Department of Research and Studies of the Saudi Food and Drug Authority.

\section{Conflict of Interest Statement}

The authors have no conflicts of interest to declare.

\section{Funding Sources}

This study was conducted by the Saudi Food and Drug Authority. There was no funding by any external organization or institution. All authors are employees of the Saudi Food and Drug Authority.

\section{Author Contributions}

The following are the authors' contribution: Salwa M. Almomen (primary author): literature review, data cleaning and analysis, manuscript writing; Mona A. Almaghrabi (co-author): literature review, data collection and cleaning, and manuscript reviewing; Saja M. Alhabardi (co-author): literature review, data collection and cleaning, and manuscript reviewing; Adel A. Alrwisan (supervisor): study design, data analysis, and manuscript reviewing.

\section{Data Availability Statement}

Data are publicly available by the United States Food and Drug Administration.
Almomen/Almaghrabi/Alhabardi/ Alrwisan 


\section{References}

1 Suk KT, Kim DJ. Drug-induced liver injury: present and future. Clin Mol Hepatol. 2012; 18(3):249.

2 United States Food and Drug Administration. Fda.gov. 2020. 2020. Available from: https:// www.fda.gov/media/116737/download Accessed 2020 Nov 1.

3 Ostapowicz G, Fontana RJ, Schiødt FV, Larson A, Davern TJ, Han SH, et al. Results of a Prospective Study of Acute Liver Failure at 17 Tertiary Care Centers in the United States. Ann Intern Med. 2002;137(12):947.

$4 \mathrm{Xu} \mathrm{JJ}$, Diaz D, O'Brien PJ. Applications of cytotoxicity assays and pre-lethal mechanistic assays for assessment of human hepatotoxicity potential. Chem Biol Interact. 2004;150(1): $115-28$.
5 Larson A. Drug-induced liver injury. Uptodate; 2020. Available from: https://www.uptodate.com/contents/drug induced-liver-injury Accessed 2020 Nov 1.

6 Temple R. Hy's law: predicting serious hepatotoxicity. Pharmacoepidemiol Drug Saf. 2006;15(4):241-3.

7 Senior JR. How can "Hy's law" help the clinician? Pharmacoepidemiol Drug Saf. 2006; 15(4):235-9.

8 Senior JR. Unintended hepatic adverse events associated with cancer chemotherapy. Toxicol Pathol. 2010;38(1):142-7.

9 United States Food and Drug Administration. Drug-induced liver injury premarketing clinical evaluation. 2020. Available from: https:// www.fda.gov/regulatory-information/ search-fda-guidance-documents/drug-induced-liver-injury-premarketing-clinicalevaluation Accessed 2020 Nov 1.
10 United States Food and Drug Administration. Accelerated-approval program. 2020. Available from: https://www.fda.gov/drugs/information-healthcare-professionals-drugs/accelerated-approval program Accessed 2020 Nov 1.

11 Sarges P, Steinberg JM, Lewis JH. Drug-induced liver injury: highlights from a review of the 2015 literature. Drug Saf. 2016;39(9):80121.

12 Downing NS, Shah ND, Aminawung JA, Pease AM, Zeitoun JD, Krumholz HM, et al. Postmarket safety events among novel therapeutics approved by the US Food and Drug Administration between 2001 and 2010 . JAMA. 2017;317(18): 1854.

13 Mosedale M, Watkins PB. Drug-induced liver injury: advances in mechanistic understanding that will inform risk management. Clin Pharmacol Ther. 2017;101(4):469-80. 